\title{
Reference interferometer using a semiconductor laser/LED reference source in a cryogenic Fourier-transform spectrometer
}

\author{
Anthony J. Martino and Donald M. Conwell \\ Lasers and Electro-Optics Branch, Code 554, NASA Goddard Space Flight Center, Greenhelt, MD 20771
}

\begin{abstract}
A combination of a single mode AIGaAs laser diode and a broadbind LED was used in a Michelson interferometer to provide reference signals in a Fourier transform spectrometer, the Composite Infrared Spectrometer, on the Cassini mission to Saturn. The narrowband light from the laser produced continuous fringes throughout the travel of the interferometer, which were used to control the velocity of the scan mechanism and to trigger data sampling. The broadbind light from the LED produced a burst of fringes at zero path difference, which was used as a fixed position reterence. The system, including the sources, the interferometer, and the detectors, was designed to work both at room temperature and at the instrument operating temperature of $170 \mathrm{Kelvin}$. One major challenge that was overcome was preservation, from room temperature to $170 \mathrm{~K}$, of alignment sufficient for high modulation of fringes from the broadband source. Another was the shift of the source spectra about $30 \mathrm{~nm}$ toward shorter wavelengths upon cooldown.
\end{abstract}

Keywords: Fourier transform spectrometer, laser diode, LED, Cassini, CIRS, reference interferometer

\section{INTRODUCTION}

The Composite Infrared Spectrometer ${ }^{1,2}$ (CIRS) for the Cassini mission to Saturn is a Fourier transform infrared spectrometer that contains three Michelson-type interferometers. One is used for spectral measurements in the mid-infinred (MIR) band of 7 to $17 \mu \mathrm{m}$; another is used in the far-infrared (FIR) band of $17 \mathrm{to} 1000 \mu \mathrm{m}$. The moving elements of these two interferometers are mounted on a common scan mechanism ${ }^{3}$.

The third interferometer, called the reference interferometer (RI), is the subject of this paper. In a Fourier transform spectrometer, it is essential to have precise knowledge of the position of the moving element as it moves. In CIRS, the reference interferometer provides this information. It is also a Michelson-type interferometer, with one of its arms terminated on the same scan mechanism as the MIR and FIR interferometers. The signals produced by the RI are used in two ways: as feedback to the circuits that control motion of the scan mechanism, and as a trigger signal for the sampling of data from the detectors in the MIR and FIR interferometers.

\section{DESIGN OVERVIEIV}

The RI is a Michelson interferometer with cube-conter retroretlectors at the ends of its arms. (See Figure 1). Two light sources, a single-frequency laser diode and at wide-spectrum LED, emit light into the RI. Their outputs are combined with a polarizing beamsplitter and collimated, and a quauter-waive plate is used for isolation.

The collimated beans are sent into the interferometer, where they are split by a nonpoliuizing beamsplitter. Both :urns itre terminated by retroreflectors; the moving retroreflector is actually the centrid portion of the element that is used in the MIR interferometer. The other retroreflector is tixed to the splical bench.

The output beams from the interferometer are split, filtered, and ficused onto pinholes to separate the laser light from the LED light. Behind each pinhole is a photodetector. 


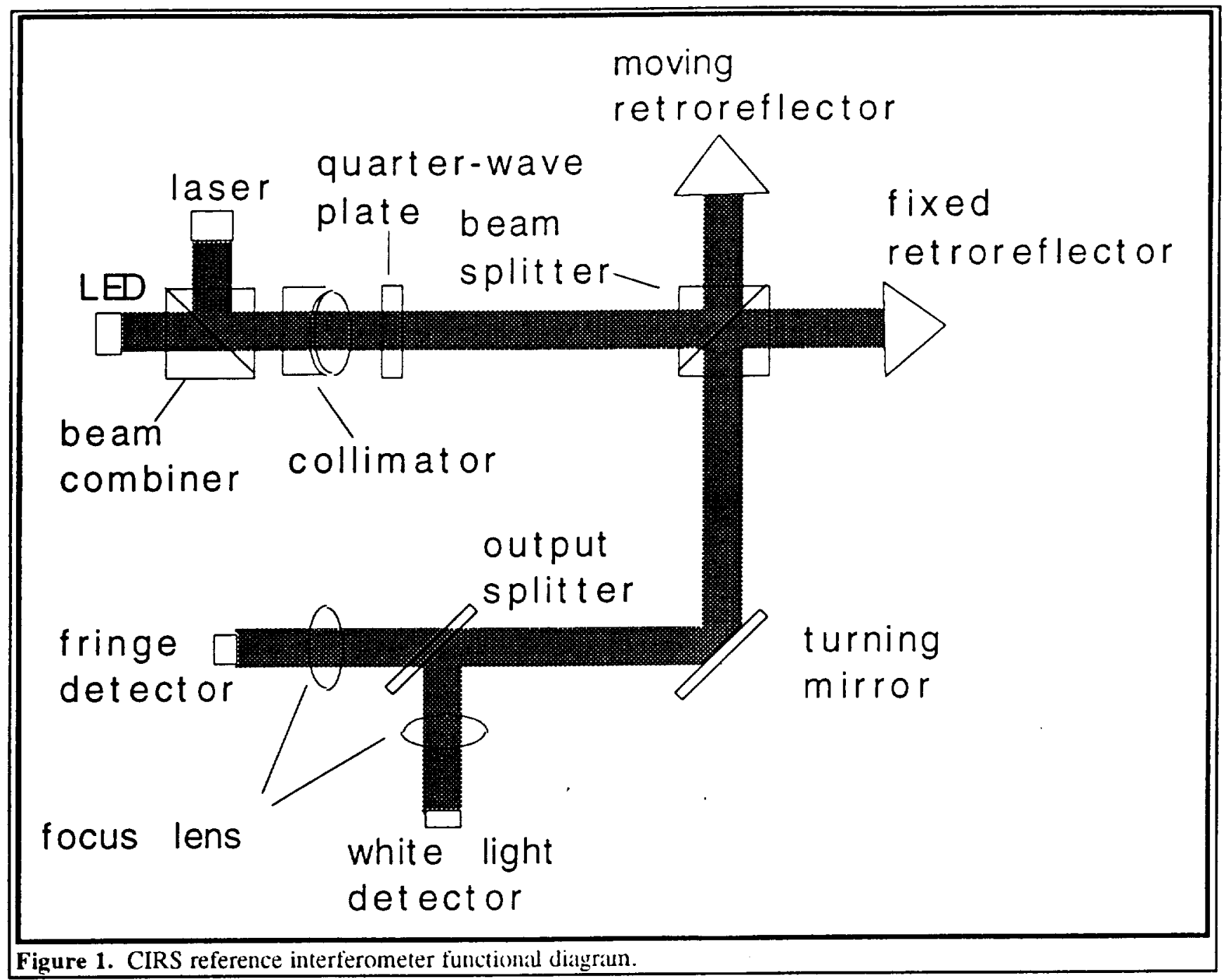

The signal from the "fringe" detector, which is produced by the laser light. consists of a sinusoid of constant aunplitude, which goes through one cycle as the scan mechamism moves half the wavelength of the laser light. The reference interferometer electronics (RIE) detects the positive and negative zero-crossings of this signal and converts them to a digital pulse train. The scan mechanism electronics lock this pulse train to a fixed frequency, causing the scan mechanism to move at a constant speed.

The signal from the "white light" detector consists of a tlat line most of the time, with a burst occurring as the RI passes through zero path difference (ZPD). The hurst consists of about 12 sinusvidlal cycles under a roughly Gaussian envelope. The period of the sinusoid is half the mean wavelength of the LED, and the highest peak is at ZPD. When the RIE detects the highest peak, it begins to send the fringe pulse train to the front end electronics, where it is used to trigger saunpling of data from the scientific focal planes.

\section{SOURCES}

In the previous instruments, ${ }^{4,6}$ for long-term space missions, on which the original design tor CIRS was batsed, neon gats discharge lamps were used as reference sources. The neon spectrum contidus a few discrete, easily separable lines, any one of which can be used to create a continuous series of fringes over a few millineters of palh length difference. It also contains a broadband "continuum" that can be used to create "white light" fringes. 
In a fiew odter spacebone fourier transform instraments, in which power and thermal dissipation were less of an issue than in

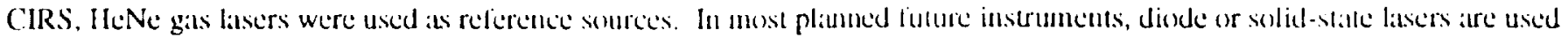

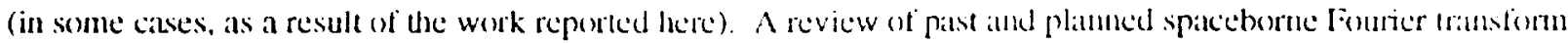
spectrometers has been published by Persky?.

During the development of CIRS, it was discovered that neon lamps were not up to de increased demands presented by CIRS. Problems were encountered in several areas: lifetime verilication, signal sucughl, and behavior of the lamps at cryogenic temperatures.

The nominal lifetimes of commercially-available neon indicator lamps, piuticuliuly when operated at DC, were significantly less that the minimum requirement of 26,000 ) hours for CIRS. Therefore. in the basieline design, two liunps were provided. The lamps would be run at a reduced current in order to extend their lifetime. A series of tests was conducted on several lamp models to verify the relation between drive current and lifetine, and to verify by accelerated test that the linns would meet the required lifetime. The test failed to verify the relation between current and lifetime given in the manutacturers' data sheets; in fact it did not yield any usable relation. Therefore, since the duration of the test was only slightly more than 1000 hours, it was inconclusive with respect to overall the lifetime requirement.

The issue of signal strength was more immediately apparent. The light emission from the neon lamps was spread through a glow discharge region approximately $1 \mathrm{~mm} \times 1 \mathrm{~mm}$. Using a hield of view of $330 \mu \mathrm{m}$ on the launp, it was possible to get an adequate signal-to-noise ratio in fringe channel over the entire range of trivel when the interferometer was perfectly aligned. With the same field of view, it was also possible to get an adequate signal-(o-noise ratio in the white-light channel at ZPD under the same conditions. However, sensitivity of signal modulation (1) misadignment increases as the field of view is endarged. ${ }^{8,9}$ When the interferometer was cooled from room temperature to its recuired operating temperature of $170 \mathrm{~K}$, it suffered sufficient misalignment that the resulting signals were useless. While the optical power incident on the detectors decreased only slightly, fringe modulation degraded so badly that the fringe pattem was often lost in the detector noise.

An even more serious problem also occurred with the trausition to cryogenic temperatures. This was the tendency of the plasma discharge within the lamps to move as a function of temperatture, and eventually to become unstable. "Flickering", that is, rapid motion of the discharge between two positions, occurred in almost every lamp below about $220 \mathrm{~K}$. While the spatially integrated output of the lamp remained relatively constant, the signal from a detector looking at a fixed field of view showed very large jumps as the discharge switched position. Finge and white light signals produced under these conditions were not usable, even if their signal-to-noise ratioss were otherwise acceptable.

As a result of these problems and uncertainties, the neon lamp design was abaudoned in favor of a semiconductor diode light source. This source consisted of a laser diode and a LED, combined opticelly. Bodh devices emitted far more light than the neon lamp from a smaller emitting area. This allowed a very strong signal w be collected from a much smaller field of view, reducing the sensitivity to adignment. In addition, both had proven lifetimes, and we were able to show that they had no objectionable behavior at $170 \mathrm{~K}$

The laser chosen for this instrument is a moditied version of the SDL 560$)^{\prime \prime \prime}$. It is mounted to all aluminum structure with a thin Delrin bushing to provide electrical insulation. A single-freyuency Fabry-Perot laser diode, it emits at about 810 nm at room temperature and $783 \mathrm{~mm}$ at $170 \mathrm{~K}$. Highly linearly poluvized light is emitted from an areat approximately $1 \times 3 \mu \mathrm{m}$ into a cone approximately $10^{\circ} \times 30^{\circ}$. Each device hats two emilling arcils, spaced about $14 \mu \mathrm{m}$ apiut, that can be controlled independently. Between mode hops, the laser exhibits a lemperature coetlicient of $0.08 \mathrm{mn} / \mathrm{K}$. Since the laser wavelength stability requirement is 1 part in $10^{5}$ over a 2 -minute time interval, it is necessiry to stibilize the temperitture to prevent excursions of more than $0.01 \mathrm{~K}$ in that time. The structure in which the $\mathrm{RI}$ is inounted is controlled to $\pm\left(0.1^{\prime \prime} \mathrm{C}\right.$ in 24 hours, and its thermal inertia is sufficient to prevent extremely rapid lemperature chamges.

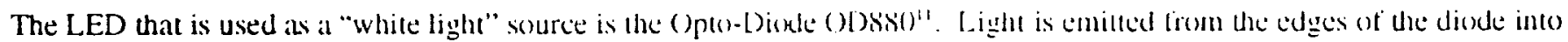
a well in the bottom of the cesse: it is the diffuse surlate of the well that serves as the effective light source for the

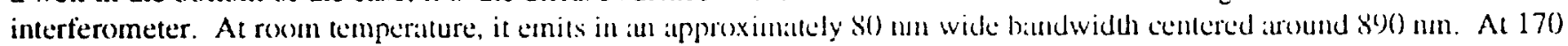
$\mathrm{K}$, the bandwidth narrows somewhat, and the lotad power approximalely doubles lor a given drive current (see Figure 2). 


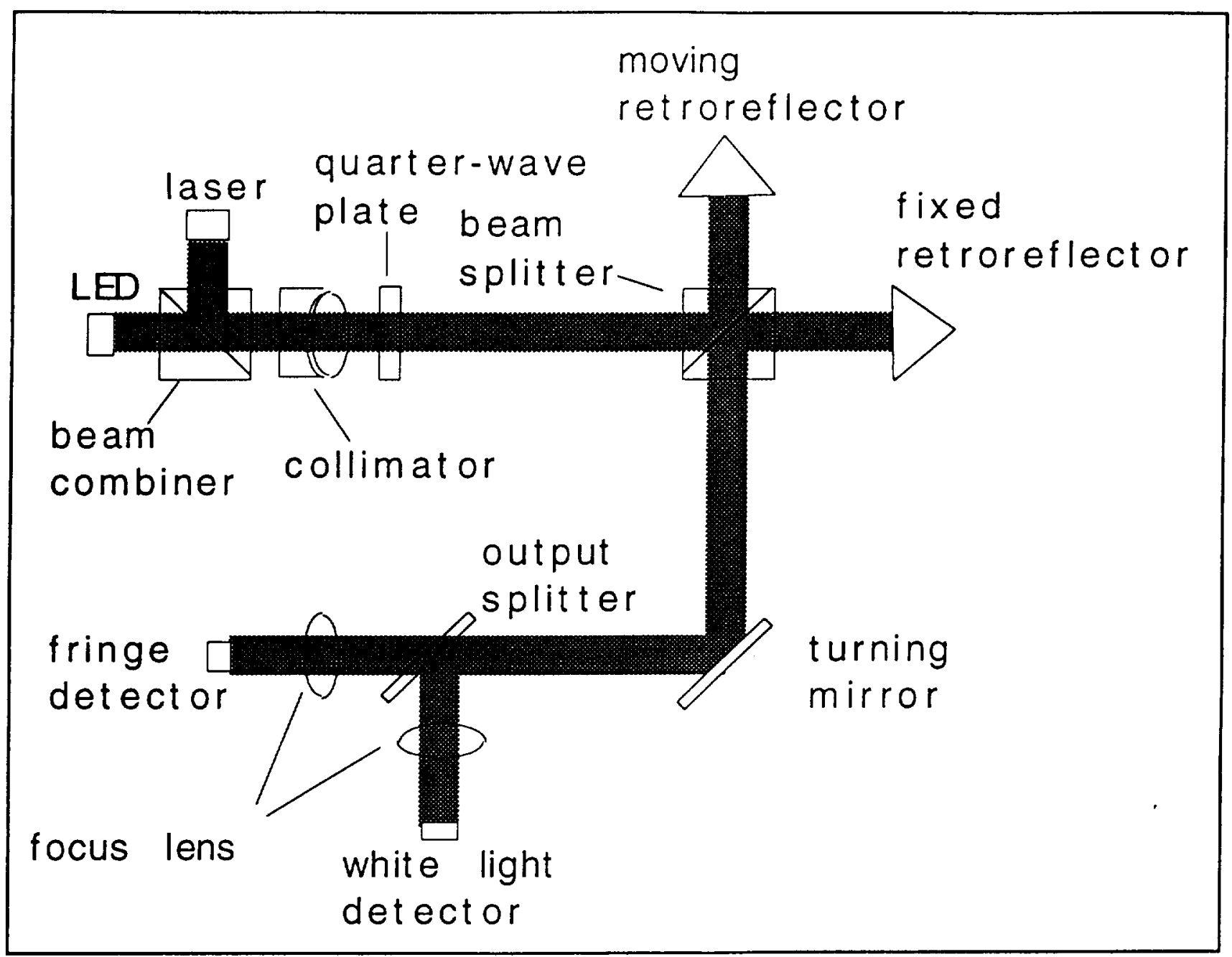

Figure 1. CIRS reference interferometer functional diagram.

The signal from the "fringe" detector, which is produced by the lisser light, consists of a sinusoid of constant amplitude, which goes through one cycle as the scan mechamism moves half the wavelength of the laser light. The reference interferometer electronics (RIE) detects the positive and negative zero-crossings of this signal and converts them to a digital pulse train. The scan mechanism electronics lock this pulse train to a fixed frequency, causing the scan mechanism to move at a constant speed.

The signal from the "white light" detector consists of a flat line inost of the time, with a burst occurring as the RI passes through zero path difference (ZPD). The burst consists of about 12 sinusoidid cycles under a roughly Gaussian envelope. The period of the sinusoid is half the mean wavelength of the LED, and the highest peak is at ZPD. When the RIE detects the highest peak, it begins to send the fringe pulse tritin to the front end clectronics, where it is used to trigger sampling of data from the scientific focal planes.

\section{SOURCES}

In the previous instruments ${ }^{45,6}$ for long-term space misvions, on which the original design for CIRS was based, neon gas discharge lamps were used as reference sources. The neon specuun contians a tew discrete, easily separable lines, any one of which can be used to create a continuosus series of lingess over at fiew millimeters of path length difference. It adso contains a broadband "continuurn" that can be used to crteile "white light" tringes. 
In a tew other spacebone Fourier trassform instruments, in which power and thermal dissipation were less of an issue than in CIRS, HeNe gas lasers were used as reterence sourees. [n most planned luture instruments, diode or solid-stale latsers are used (in some calses, as a result of due work reported bere). A review of past and platumed spacehone Fourier transfonn spectometers hiss heen published by Persky'.

During the development of CIRS, it was discovered hat nern limps were not up to dhe increatsed demands presented by CIRS. Problems were encountered in several areats: lifetime verificalion, sighal stuenglh, aud belhavior of the lamps at cryogenic temperatures.

The nominal lifetimes of commercially-available neon indicator lanps, piuticulauly when operated at DC, were significantly less that the minimum requirement of 26,000 hours for CIRS. Therefore, in the balieline design, two launps were provided. The lamps would be run at a reduced current in order to extend their lifetime. A series of tests was conducted on several lamp models to verify the relation between drive current and lifetine, and to verify by accelerated test that the liunps would meet the required lifetime. The test failed to verify the relation between current and lifetime given in the inanufacturers' data sheets; in fact it did not yield any usable relation. Therefore, since the duration of the test wats only slightly more than 1000 hours, it was inconclusive with respect to overall the lifetime requirement.

The issue of signal strength was more immediately appiuent. The light emission from the neon lamps was spread through a glow discharge region approximately $1 \mathrm{~mm} \times 1 \mathrm{~mm}$. Using a tield of view of $330 \mu \mathrm{m}$ on the lamp, it was possible to get an adequate signal-to-noise ratio in fringe channel over the entire runge of travel when the interferometer was pertectly aligned. With the same field of view, it was also possible to get an adequate signal-to-noise ratio in the white-light channel at ZPD under the same conditions. However, sensitivity of signal modulation to misalignment increases as the field of view is enlarged ${ }^{8.9}$ When the interferometer was coled from room temperature to its required operating temperature of $170 \mathrm{~K}$, it suffered sufficient misalignment that the resulting signals were useless. While the optical power incident on the detectors decreased only slightly, fringe modulation degraded so badly that the fringe pattem was often lost in the detector noise.

An even more serious problem also occurred with the trinsition to cryogenic temperatures. This was the tendency of the plasma discharge within the lamps to move as a function of temperature, and eventually to become unstable. "Flickering", that is, rapid motion of the discharge between two positions, occurred in almost every launp below about $220 \mathrm{~K}$. While the spatially integrated output of the lamp remained relitively constant, the signal from a detector looking at a fixed field of view showed very large jumps as the discharge switched position. Fringe and white light signals produced under these conditions were not usable, even if their sigmal-to-noise ratios were otherwise acceptable.

As a result of these problems and uncertainties, the neon liump design was abandoned in favor of a semiconductor diode light source. This source consisted of a laser diode and a LED, combined optically. Both devices emitted far more light than the neon lamp from a smaller emitting area. This allowed a very strong signal to be collected from a much smaller field of view, reducing the sensitivity to alignment. In addition, both had proven lifetimes, and we were able to show that they had no objectionable behavior at $170 \mathrm{~K}$.

The laser chosen for this instrument is a inoditied version of the SDL 560$) 1^{117}$. It is inounted to an aluminum structure with a thin Delrin bushing to provide electrical insulation. A single-frequency Fabry-Perot laser diode, it emits at about 810 nm at room temperature and $783 \mathrm{~mm}$ at $170 \mathrm{~K}$. Highly linciuly polivized light is cmitted from an auca approximately $1 \times 3 \mu m$ into a

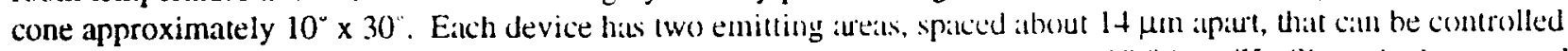
independently. Between mode hops, the latser exhibits a temperature cocticicient of (0.08 miK/ Since dhe latser wavelength stability requirement is 1 part in $10^{5}$ over a 2 -minute time interval, it is necessiry to stubilize the temperature to prevent excursions of more than $0.01 \mathrm{~K}$ in that time. The structure in which the RI is incounted is controlled $10 \pm 0.1^{\circ} \mathrm{C}$ in 24 hours, and its thermal inertia is sufficient to prevent excremely ropid temperatture changes.

The LED that is used as a "white light" source is the (Opto-Diode (ODsso". Light is emilted tiom the edgets of the diode into a well in the botkom of the case; it is the diffuse surlike of the well thall serves as the clfective light source tor the interferometer. At room temperature, it emits in an approximately 80 mn wide bandwidu centered around 800 mm. At 170 $\mathrm{K}$, the bandwidth narrows somewhat. and the kotal power appoximately doubles lor a given drive curcent (see Figure 2). 


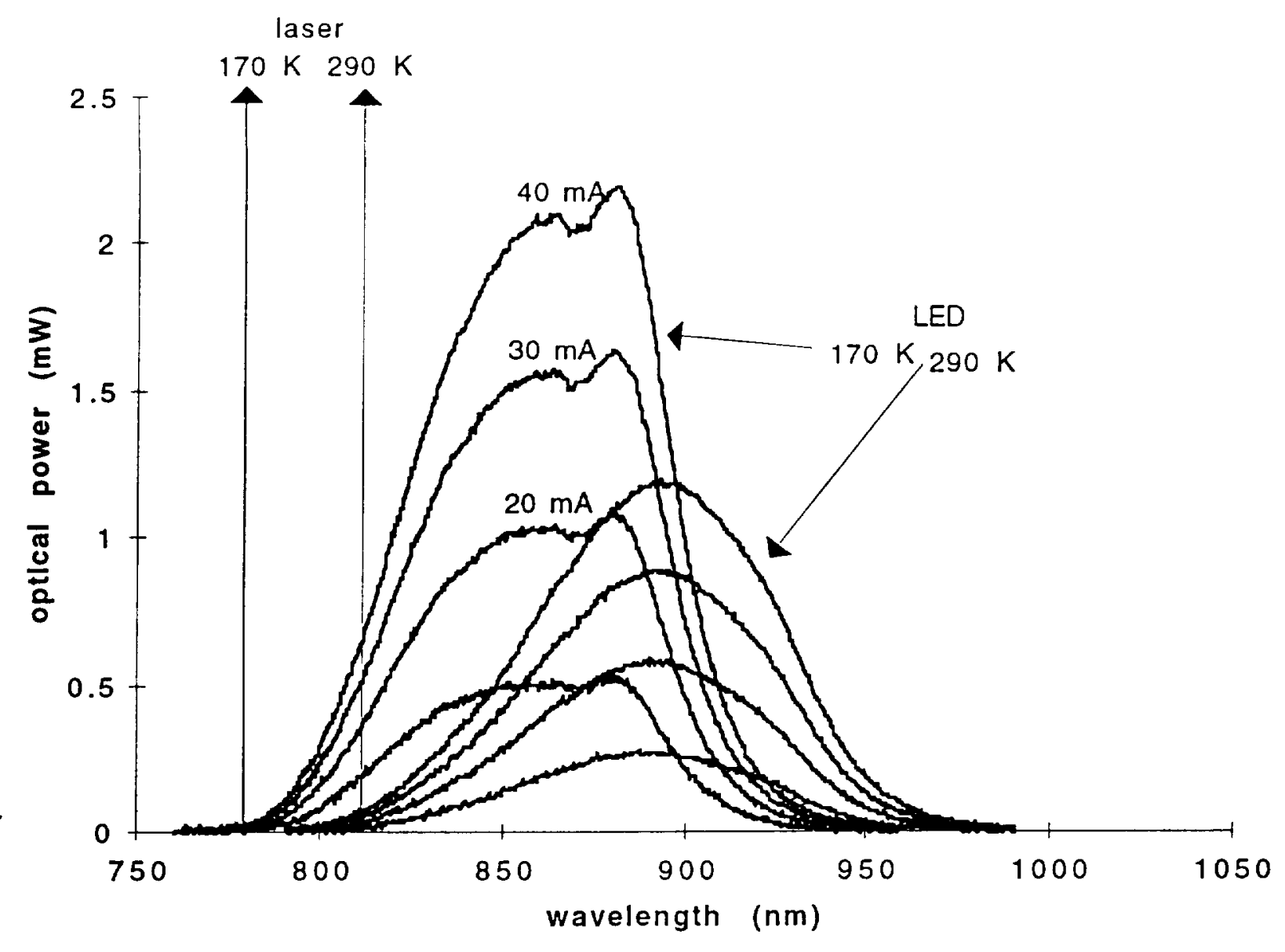

Figure 2. Dependence of source spectra on temperature and drive current

\section{OPTICS}

The optics can be divided into three sections: the source optics, which form the beams; the interferometer optics, which modulate the beams; and the receiver optics, which separate the lisser and LED beams and focus them onto their respective detectors.

The lenses and beam splitters and combiners are all made from two radiation-resistimt glass types: Schott BK7-G25 and LF5G15. The instrument is required to withstand up to 100 krad, mostly from high-energy protons.

\subsection{Source optics}

The LED is placed with its center on the optical ixis of the interferometer. Half of its randomly-polarized light is transmitted by the polarizing beam combiner. It is collimated by the $28 \mathrm{~mm}$ fical length doublet into a $9 \mathrm{~mm}$ diameter beam, which is sent into the interferometer.

The difference in power output between the laser and the LED is so liarge that spectral tiltering alone would not be sufficient to separate the two beams at the output of the interferometer. In addition, the retrorellectors in the interferometer would send a large portion of the laser light back to its source, creating severe instibility in the frequency and power output of the laser. These problems were alleviated by putting the LED on axis and the laser off axis and introducing an input aperture into the laser's optical path. (See Figure 3.) The spatial sepasaltion hetween lhe laser and the LED is repeated in the innage plane at the output of the system, so the "white light" delectur sces only scittered light from the lister. The laser input aperture 
reduces the anount of laser light that enters the interferometer. Also, it caluses ath of the latser light to hit the retrorethectors on one side of eenter. The returned be:un is on the other side of center, and decrefore misses the aperture and does mot hit the laser.

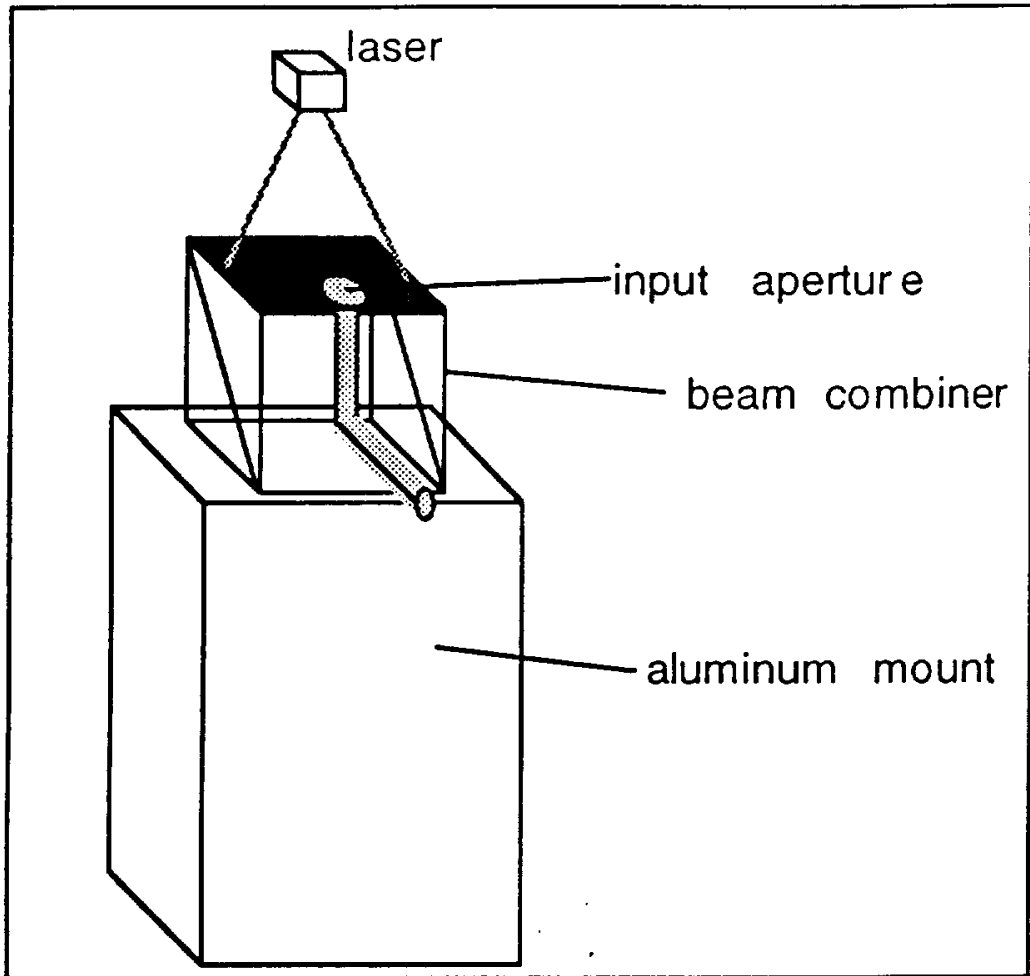

Figure 3. Laser input optics
The laser input apcrture and the be:un combiner mount are made of aluminum. They are bonded to the glass bean combiner with RTV $566^{12}$, a silicone rubber adhesive, after preparing the surfices with $S S+155$ primer. While the resulting bond is strong enough to withstand the vibration of launch, it is still compliant enough to prevent breakage due to differential contraction between the glass and aluminum as the instrument is cooled from room temperature to $170 \mathrm{~K}$.

While the input aperture effectively prevents the retroreflectors from causing feedback into the laser, it does nothing to prevent feedback from flat surfaces perpendicular to the bean. While reflections from these surfaces are minimized by antireflection coatings, an additional measure of protection is obtained by using a crystalline quartz cuarter-wave plate after the collimator. The laser is oriented so that its light is reflected by the polarizing beam combiner. The quarter-wave plate has its axis at $45^{\circ}$ to the polarization of the incident laser light. Light that is reflected back from perpendicular surfaces in the interferometer has its polarization rotated $90^{\circ}$ after the second pass through the quarter-wave plate. Rather than being reflected back into the laser by the becun combiner, it is transmitted harmlessly onto the LED.

\subsection{Interferometer optics}

The laser and LED beams follow a common path through the interferometer. They are split by a nonpolarizing cube beamsplitter. The reflected beam is retumed from the moving retroreflector, and the transmilted bean is returned from the fixed retroreflector. The returned beams are recombined at the beamsplitter, then directed by a fold mirror to the receiver.

Preservation of alignment between the beamsplitter and the two retroreflectors during the transition from room temperature to $170 \mathrm{~K}$ is vital to the performance of the interferometer. In a Michelson interferometer that uses cube comer retroreflectors, the critical alignment is shear between the two beiuns. This can be affected by tilt of the beiunsplitter and translation of the cube corners.

The cube beamsplitter was fabricated with a cylindrical extension on the ficce towitud the fold mirror. The extension is inserted in one end of a cylindrical portion of the interferometer structure, and the fold mirror is inserted in the ofler end. (See Figure 4.) RTV 566 is injected through small holes in the aluminum cylinder to hold the optics in place. In earlier versions, the cylinder was a separate piece, bonded with RTV $566 \mathrm{w}$ the rest of the structure. This arrungement did not maintain alignment well enough on conldown. In the flight unit, the entire structure ("interterometer housing") shown in Figure + is a single piece of aluminum, which is bolted into the CIRS opticid bench. The source and receiver palckigess are bolled onto the interferometer housing.

The moving and fixed retrorellectors are glass hollow cube coners, mide at Goddlard Space Flight Center ${ }^{1.14}$. The moving reflector is shared with the MIR interferoneter and is mounted on the sciul mechanism. The MIR interferometer uses the 


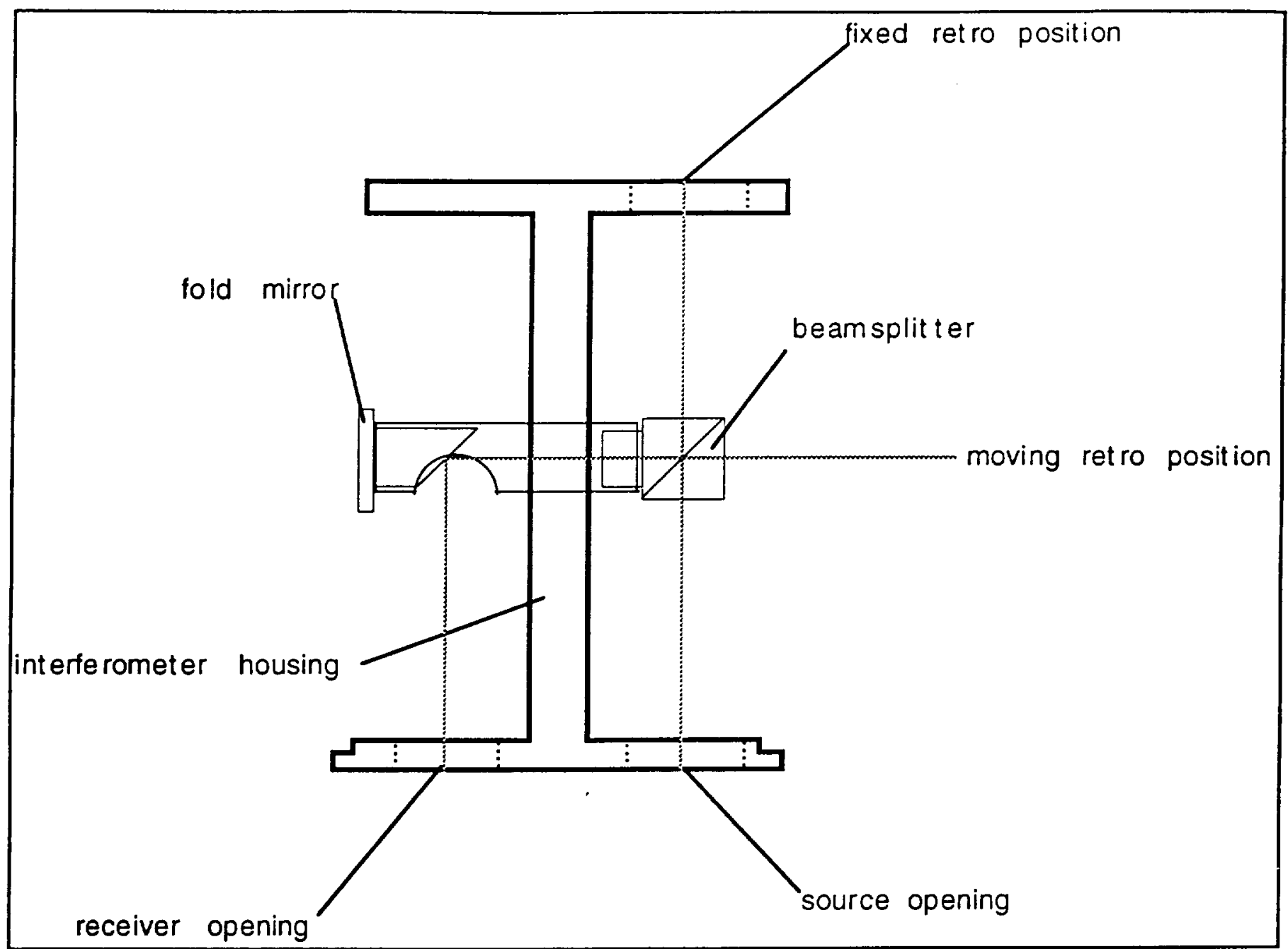

Figure 4. Interferometer structure

outer portion of the reflector, while the RI, which is located in the shadow of the telescope secondary mirror, uses the central portion. The fixed reflector is mounted on the optical bench.

The fold mirror was diamond-tumed at $45^{\circ}$ to the axis of an aluminum cylinder at Marshall Space Flight Center, then coated with gold at Godklard.

\subsection{Receiver optics}

The receiver consists of two channels. The "fringe" signid is detected in one chamnel, and the "white light" signal is detected in the other. Light that comes from the interferometer is divided between the two channels by a neutral beamsplitter, which reflects $90 \%$ of the light into the "white light" channel and triusmits $10 \%$ into the "fringe" channel.

In each channel, the desired signal is separated from the other signad, and from stray light, by spectral and spatial filtering. A $28 \mathrm{~mm}$ focal length singlet lens focuses the collimated beims, forming a unit-magnification image of the sources at its focal plane. The "fringe" lens is coated with a multi-layer interference coating that is designed to pass the laser wavelength but block the LED spectrum at $170 \mathrm{~K}$. The "white light" focus lens has a corting that does the opposite. Because of the close spacing between the laser and LED wavelenguss (see Figure 2), it is no possible to design a coating that cleanly separates the two spectra at both room temperature and $170 \mathrm{~K}$, so some crosst:dk is untwoidible at room temperature. 
A $150 \mu \mathrm{m}$ diancter circular ficld stop is located in the focal plane of each leus. The "fringe" field stop, which is centered on the image of the laser, serves inostly to block striy light. since the innlge of the laser is smaller than the aperture dianeter. The "white light" field stop, which is centered on the image of the LED, actually limits the areal of the image that is "seen" by the detector. This is necessiry to limit the sensitivity of the signad to shear.

Because of the large difference in power hetween the lister and LED, the blocking performance of the "white lighe" interference filter is only good enough to prevent crosstalk from the peripheral regions of the laser bean. However, the laser and the LED are offset by more than the field size in the source plane, so the direct laser bein is blocked by the field stop in the "white light" focal plane.

\section{DETECTORS}

Photodetectors are located directly behind the field stops in both receiver channels. Both detectors are EG\&G SGD100 silicon PIN photodiodes ${ }^{15}$. These detectors have $2.5 \mathrm{mn}$ dianeter active areas, so they are oversized with respect to the cone of light coming through the field stop. This makes them relatively insensitive to alignment.

Each photodiode is followed by a transimpedance amplifier. The gains of the amplifiers are set to put the output signals between 0 and 10 Volts for the range of optical inputs.

\section{PERFORMANCE}

Figures 9 through 12 show examples of the fringe and white light signals produced by the RI hardware after its installation into the Cassini CIRS instrument. The data for figures 9 and 10 were measured with the instrument cooled to $170 \mathrm{~K}$ in a thermal vacuum chamber. The data for figures 11 and 12 were measured with the instrument at room temperature in air.

In general, the performance of the interferometer is better at its inteded operating temperature of $170 \mathrm{~K}$ than at room temperature. Reasons for this include greater efficiency of the light sources and better spectral properties of the laser when cold, and better separation of the channels as the spectra of the light sources shifts to the wavelengths for which the interference filters were designed.

The greater efficiency of the laser and LED means that a stronger signal is obtained for a given drive current. This effect is most evident in the white light signals. Both sets shown here were inealsured at the same drive current; the height in the cold case is twice what it is in the warm case. For the fringe signals, this is less evident, because it was necessary to run the laser at different drive currents depending on temperature. Typically, the laser drive current is reduced by about a factor of 2 between room temperature and $170 \mathrm{~K}$.

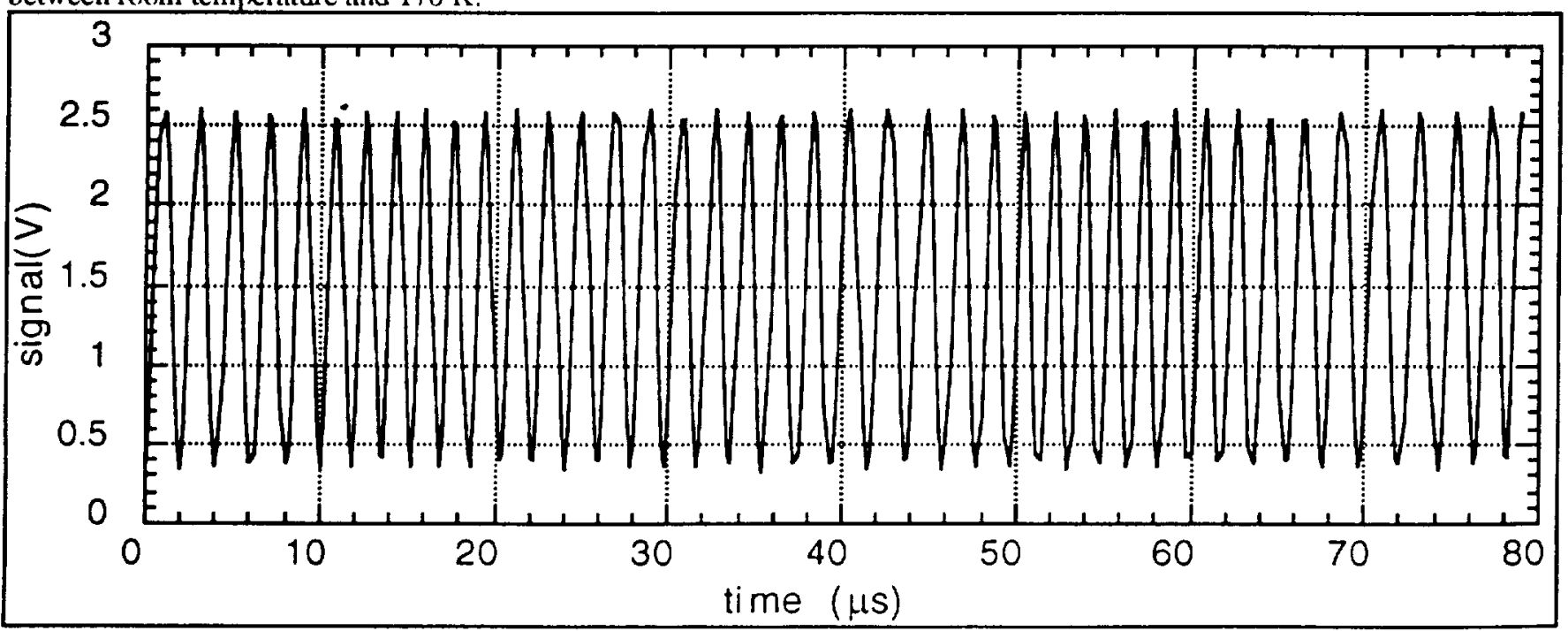

Figure 9. Fringe signal all instrument operalling temperillute of $170 \mathrm{~K}$. 


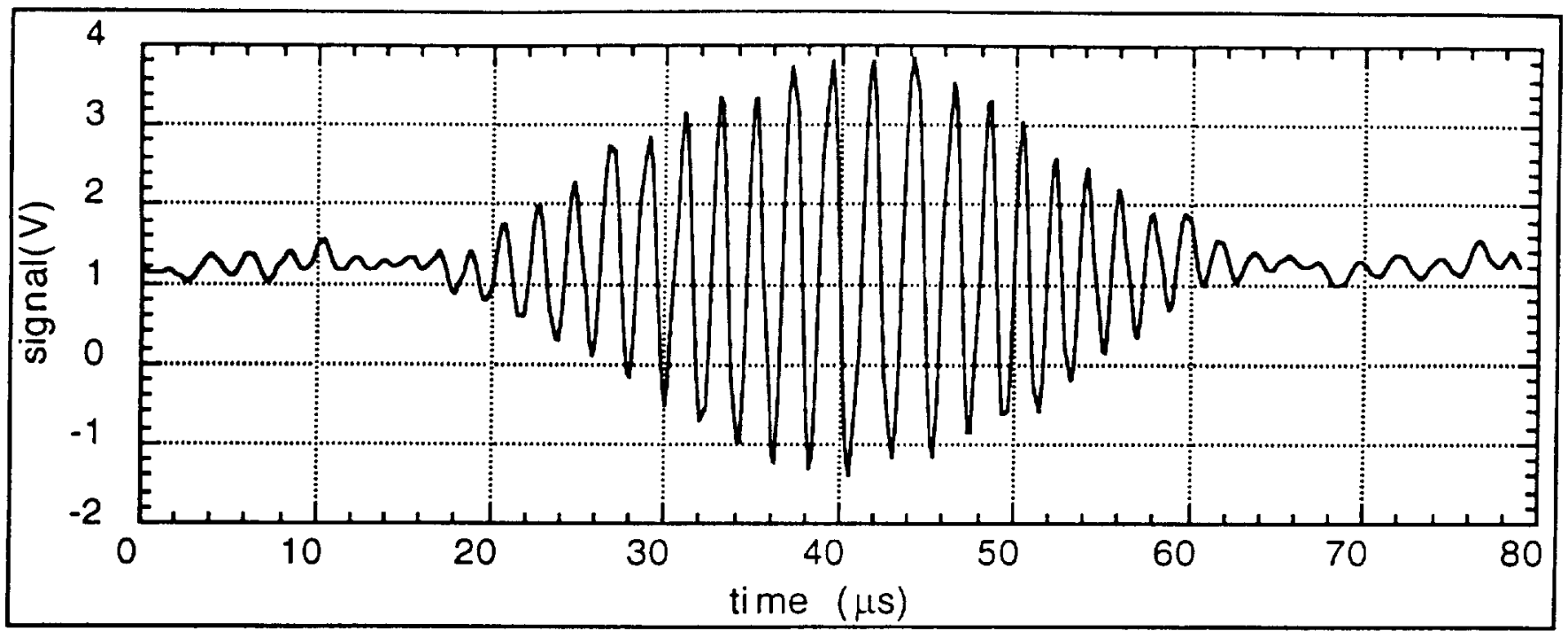

Figure 10. White light signal at instrument operating temperature of $170 \mathrm{~K}$.

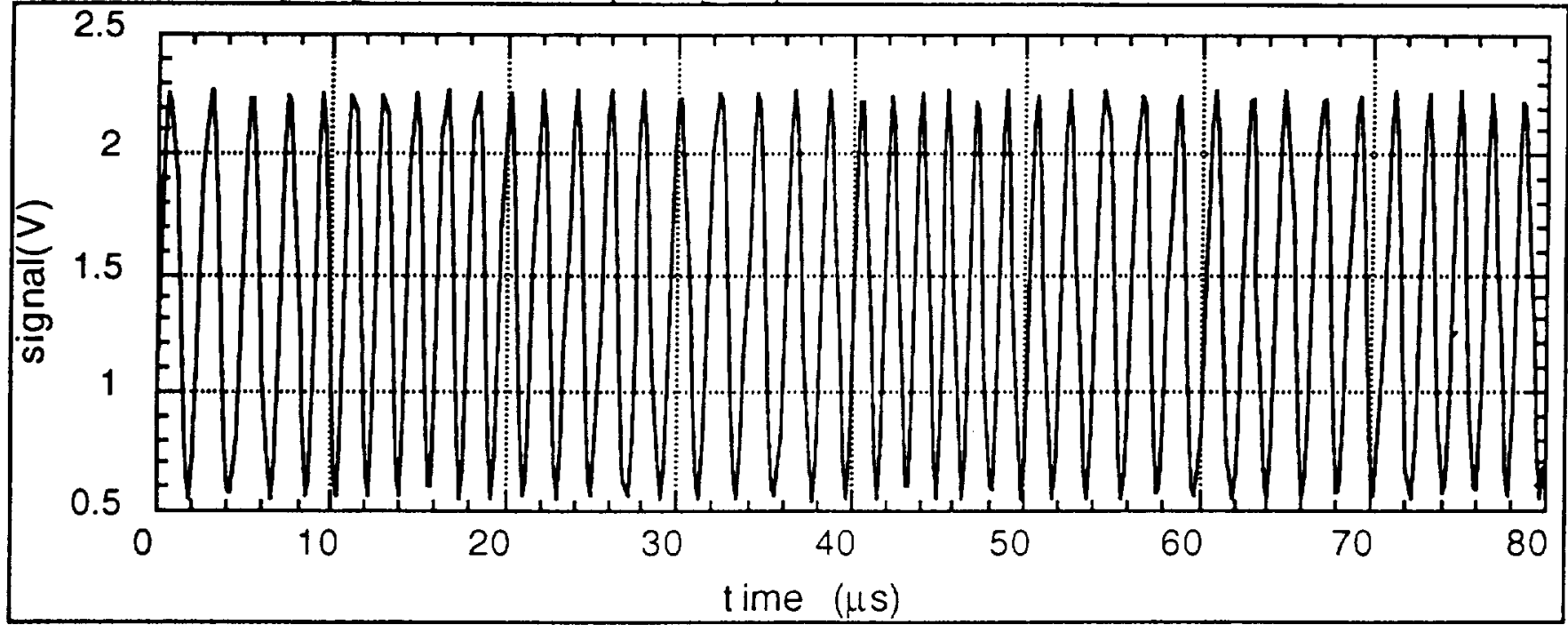

Figure 11. Fringe signal at room temperature.

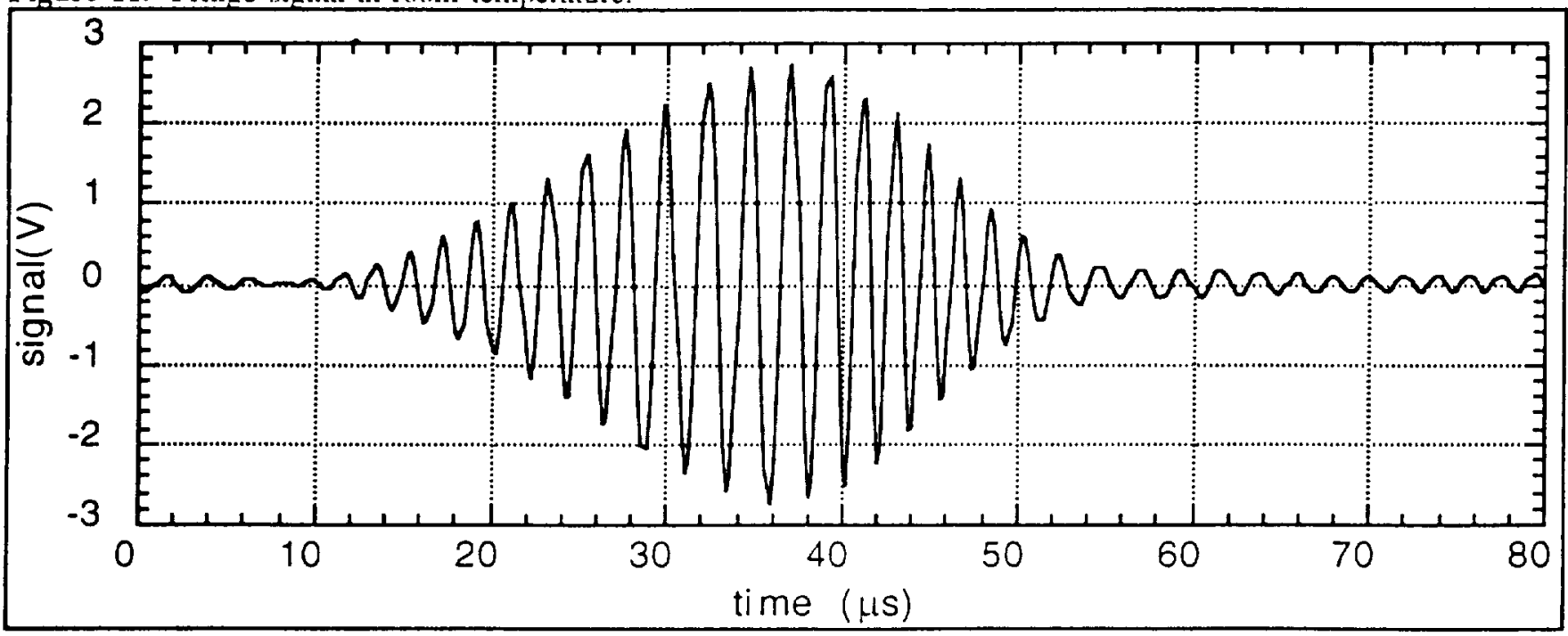

Figure 12. White light signal at romm temperature. 


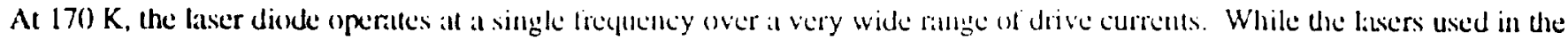

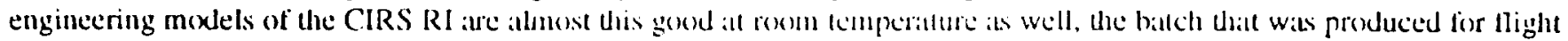
was found to be single-trequency only within a tow very natrow ranges of drive current. Simec the instrument temperature is not under control at room temperature, the laser kends to drith. Conscyuchlly, it usuatly runs mulunode. This produces a fringe height that varies over the length of de scan, Jue to beating hetween the two ficepuencies. While such behavior would

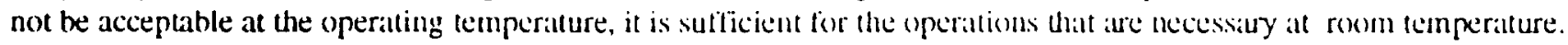

As mentioned before, the separation between the laser and LED specta is about the sane ats the ir common shilt between room temperature and $170 \mathrm{~K}$, so it is not possible $(0$ sepiualle them cleanly all hoth temperaltures with a tixed tilter.

Comparison of the warm and cold white light signads shows evidence of this. While the signal away from the white light fringes shows only noise in the cold case, it clearly shows leakage of the tringe signal in the warm caste.

The CIRS instrument, including the reference interterometer, wats launched abourd the Cassini spacecraft in October, 1997 and is now on its way to Satum. Telemetry signals have been received trom CIRS RI on several occasions since then, and all are within their nominal range. All indications are that it is working properly, giving the sane performance as before launch.

\section{REFERENCES}

1. V. Kunde et. al., "Cassini intrared Fourier spectroscopic investigation," Proc. SPIE 2803, pp. 162-177, 1996.

2. P. W. Maymon et al., "Optical design of the composite infrated spectrometer (CIRS) for the Cassini mission," Proc. SPIE 1945, pp. 100-111, 1993.

3. C. F. Hakun and K. A. Blumenstock, "A cryogenic scan mechanisin for use in Fourier tr:unsform spectrometers," in NASA Johnson Space Flight Center 29th Aerospace Mechanisms Symposium, pp. 316-333, 1995.

4. R. Hanel et al., "Infrared spectrometer for Voyager," Appl. Opt. 19, pp. 1391-1400, 1980.

5. R. Hanel et al., "Mariner 9 Michelson Interterometer," Appl. Opt. 11, pp. 2625-2634, 1972.

6. R. Hanel et al., "The Nimbus III Michelson Interferometer," Appl. Opt. 9, pp. 1767-1774, 1970.

7. M. J. Persky, “A review of spaceborne intrared Fourier transform spectrometers for remote sensing," Rev. Sci. Instrum. 66, pp. 4763-4796, 1995.

8. M. V. R. K. Murty, "Some more aspects of the Michelson interterometer with cube comers,"J. Opt. Soc. Am. 50, pp. 7$10,1960$.

9. A. J. Martino and J. G. Hagopian, "Effects of shear, defocus, and wavetront error on the theoretical performance of the composite infrared spectrometer for Cassini," Proc. SPIE 3435, in press, 1908.

10. SDL, Inc., 80 Rose Orchard Way, San Jose, CA 95134.

11. Opto-Diode Corp., 750 Mitchell Road, Newbury Park, CA 91320.

12. GE Silicones, 260 Hudson River Road, Watertord, NY 12188.

13. J. J. Lyons and P. A. Hayes, "High-optical-quality cryogenic hollow retroretleckors," Proc. SPIE 2541), Pp. 94-100, 1995.

14. P. A. Hayes, D. E. Jennings, and J. J. Lyons, "Development and testing of the Composite Intrited Spectrometer (CIRS) retroreflectors," Proc. SPIE 2227, pP. 37-43, 1094.

15. EG\&G Canada, 22001 Dumberry Road, Vaudreuil, Quchec, Camada I7V 8P7. 